\title{
Application of a Small Electrostatic Desalination Equipment
}

\author{
Huawei $\mathrm{Xu}^{1, \mathrm{a}}$ \\ ${ }^{1}$ China Datang Corporation Science and Technology Research Institute Co.,Ltd. Huazhong branch \\ a29604980@qq.com
}

Keywords: Electrostatic desalination, Electrode material, Electrode plate.

Abstract. The principle and characteristics of electrostatic desalination technology has been introduced, a small electrostatic desalination equipment has been made by myself with 10 electrode plates, and the spacing between two plates is $10 \mathrm{~mm}$. The desalination of the equipment is better when the DC voltage of the electrode plate is $2.5 \mathrm{~V}$, the flow of raw water is $20 \mathrm{~L} / \mathrm{h}$, the conductivity decreased from $85 \mu \mathrm{S} / \mathrm{cm}$ to $68 \mu \mathrm{S} / \mathrm{cm}$ in the first 35 minutes.

\section{Introduction}

With the large increase in China's industrial output value, industrial water consumption will also grow at a rapid rate. Industrial water sources are usually surface water and groundwater, and the salt content is relatively high. It usually requires softening or desalination to meet the requirements; The softening and desalting techniques include distillation, ion exchange, electrodialysis, reverse osmosis, etc, but they still have high energy consumption, membranes or ion resins are susceptible to pollution, relatively high operating technology requirements and relatively high equipment manufacturing costs, etc;Electrostatic desalination, also known as electro adsorption, is a new technique to remove of ions from water, it has been more and more people's attention and research because of its simple equipment, low energy consumption, no pollution and other characteristics.

\section{Basic principle}

The electric double layer exists at the junction of electrodes and the solution, it has the characteristic of capacitance, can charge or discharge in the electrochemical system, the charge of electrode side is provided by electron on the electrode surface or positive charge, the charge of solution side is provided by the cation or anion ${ }^{[1,2]}$. Under the condition of adding voltage but no electrochemical reaction, when the electrode is charged, the ions in the water will be enriched on the electrode. Electrostatic desalination technique is the use of the principle to adsorb ions or charged particles in the water, soluble salts and other charged substances are concentrated on the surface of the electrode to purify the water.

The raw water enters the electrode channel from one end. In the channel, the anion, cation or charged particle in the raw water is moved by the electric field force to the electrode of opposite polarity, and is eventually adsorbed by the double electric layer on the electrode surface. When the voltage is removed to discharge the double layer, the ions absorbed by the double layer are released again, so that the double layer is regenerated to prepare for the next round of electrosorption, and the ions and particles in the raw water are removed during this charge and discharge process.

\section{Electrode module production}

The electrode material is an important factor which affects the electrostatic desalination techniques, and the double layer electrode material with good performance should be provided by R.W.Pekala ${ }^{[3,4]}$ : (1) high specific surface area; (2) good conductivity; (3) good polarization ability; (4) no chemical reaction with the reaction liquid. Therefore, the development of electrostatic desalination techniques is mainly reflected in the research progress of electrode materials, which is the main research direction at home and abroad ${ }^{[5 \sim 13]}$. 
In this experiment, the activated carbon fiber with a specific surface area of $1800 \mathrm{~m}^{2} / \mathrm{g}$ was used as the electrode material, which has the advantages of large specific surface area, high conductivity, stable chemical property, good polarization performance and low price.

The thickness of $2 \mathrm{~mm}$ activated carbon fiber glues to the outer plate on both sides with conductive epoxy resin, which is the $200 \times 200 \mathrm{~mm}$ good conductivity of stainless steel plate. The upper hole of the plate is connected with a conducting wire, which is used as a single electrode plate. The two electrode plates can be made into a electrode module with different spacing ${ }^{[14 \sim 17]}$.

\section{Test results and analysis}

Test equipment. A small desalination equipment is maked with 10 of the electrode plates, the spacing between two plates is $10 \mathrm{~mm}$, the DC voltage applied to each electrode plate can be adjusted, and the raw water velocity can be adjusted. Anode plates and cathode plates are arranged alternately, which embed in rectangular plexiglass container. The raw water flows in a $\mathrm{W}$ shape desalination equipment, in order to increase the residence time and the desalting efficiency, as shown in Figure 1.

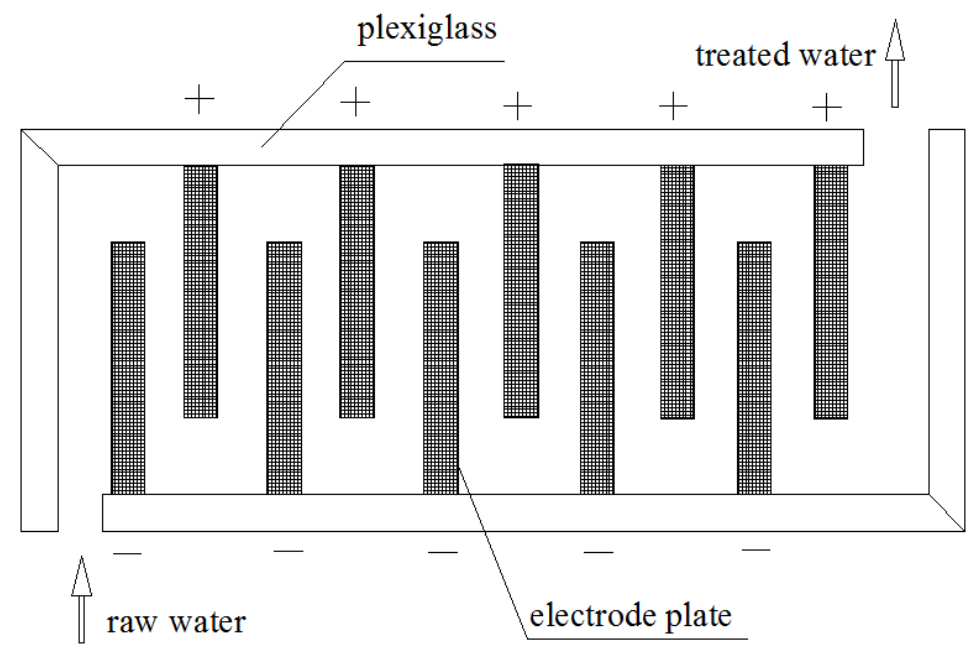

Figure 1 A small electrostatic desalination equipment

Optimal Voltage Selection. The electrical conductivity of raw water is $85 \mu \mathrm{S} / \mathrm{cm}$, and the raw water velocity is $20 \mathrm{~L} / \mathrm{h}$, in the first 35 minutes, the electrical conductivity of treated water below 68 $\mu \mathrm{S} / \mathrm{cm}$, when the voltage of electrode plate is $2.5 \mathrm{~V}$, after 1 hour, the conductivity is increased to 85 $\mu \mathrm{S} / \mathrm{cm}$, At the other two voltages $(1.5 \mathrm{~V}$ and $3.5 \mathrm{~V})$, the conductivity increased to $85 \mu \mathrm{S} / \mathrm{cm}$ at 40 minutes. Therefore, the desalination effect is best when the voltage is $2.5 \mathrm{~V}$, as shown in Figure 2.

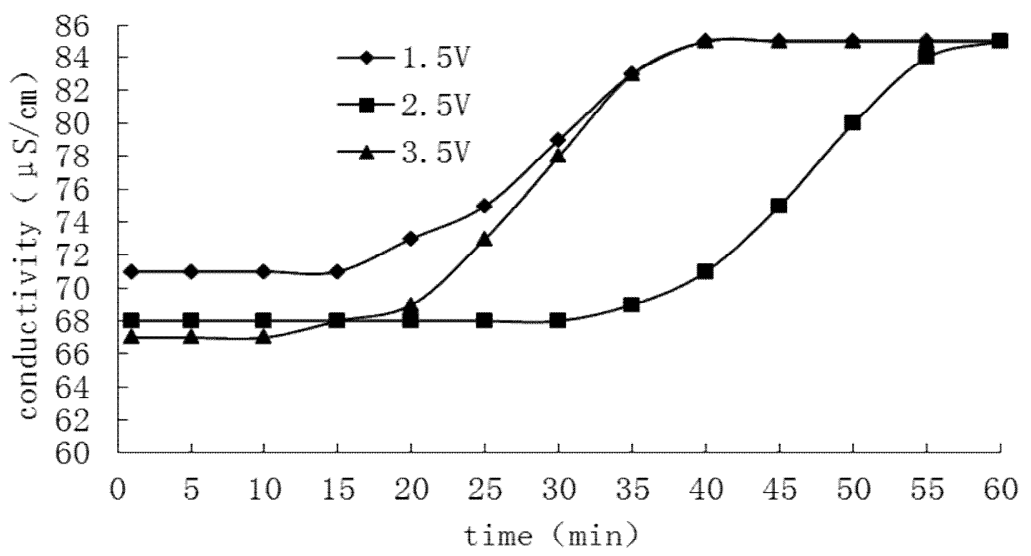

Figure 2 Voltage influence on the electrical conductivity

Optimal water flow selection. The electrical conductivity of raw water is $85 \mu \mathrm{S} / \mathrm{cm}$, The voltage of electrode plate is $2.5 \mathrm{~V}$, in the first 35 minutes, both conductivity values of treated water below 68 
$\mu \mathrm{S} / \mathrm{cm}$, and is not very different, when the influent flow rate is $15 \mathrm{~L} / \mathrm{h}$ and $20 \mathrm{~L} / \mathrm{h}$. The conductivity is $70 \mu \mathrm{S} / \mathrm{cm}$ in the first 20 minutes, then quickly rose to $85 \mu \mathrm{S} / \mathrm{cm}$. So the water flow of $20 \mathrm{~L} / \mathrm{h}$ is more efficient, as shown in Figure 3.

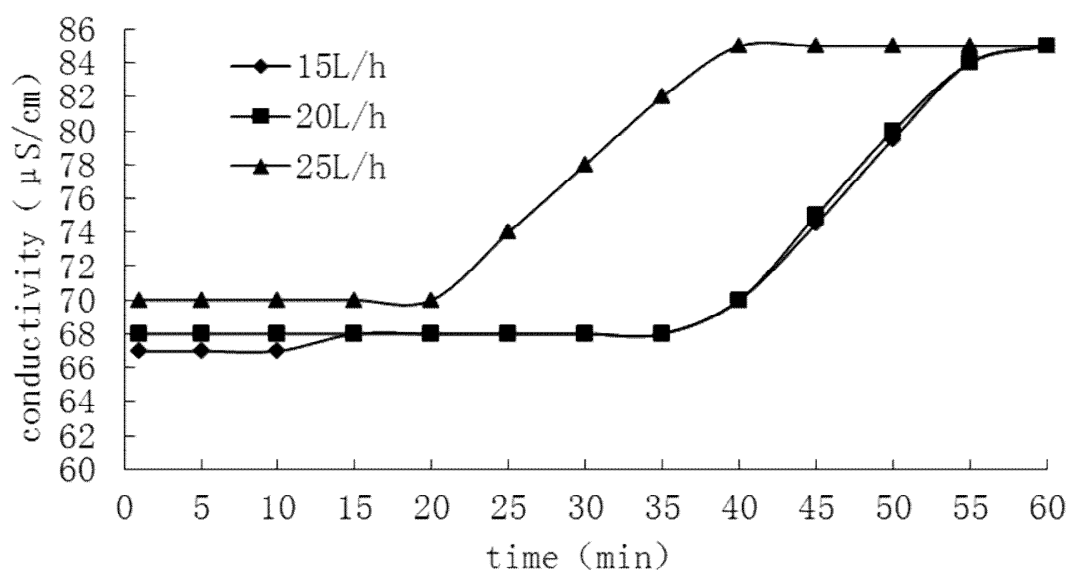

Figure 3 Water flow influence on the electrical conductivity

\section{Conclusion}

Tests have shown that the electrostatic desalination equipment made of activated carbon fiber cloth has a good desalting effect when the voltage is $2.5 \mathrm{~V}$, and the inflow flow rate is $20 \mathrm{~L} / \mathrm{h}$. When the water quality requirements are high, electrostatic desalination equipments can be connected in series. At present, the research on electrostatic desalination technology at home and abroad is mainly the research and development of electrode materials, the improvement of the adsorption performance of electrode materials, the reduction of production costs, the development of new materials, etc. will all play a catalytic role in electrostatic desalination technology.

\section{References}

[1] Cha Zhenlin, Yu Yixiong, Luo Yatian, Xu Shunhong, Li Xihe. Electrosorption technology and its application in water treatment. Sichuan Chemical Industry, 2005, 8(4): 52 54

[2] Sun Shiqiang, Cheng Jie, Cao Gaoping, Yang Yusheng. Electrostatic desalination technology research progress. Industrial Water Treatment, 2006, 26(10): 1 6

[3] Li Zhi, Zhang Yuxian. Discussion on desalting technology for flow-type capacitors [J]. Water Supply and Drainage, 2006, 32: 167-170.

[4] R.W.Pekala, J.c.Farmer, et al.Carbon aerogels for electrochemical applications .

Non-crystalline solids, $1998(225): 74 \sim 80$.

[5] Gadkaree K P, Mach J F, Stempin J L.Ion-removal fromwater using activated carbon electrodes[P].US6214204, 2001-04-10.

[6] Pekala R W.Organic aerogels : microstructural dependence of me-chanical properties in compression[J]。J.Non-cryst。Solids, 1990,125(1 2): 67〜75。

[7] J H Richardson, J C Farmer, D V Fix, et al.[C].Capacitive Deionization System, The Seventh National Technology Transfer Conference and Exposition, Anaheim, CA, 1996。

[8]Iijima S.Helical microtubules of graphitic carbon[J].Nature, 1991，354:56 58。

[9] Dai Kai, Shi Liyi, Fang Jianhui, et al. Electrosorption desalination process of carbon nanotubes electrode [J]. Journal of Applied Science, 2005,23 (5): 539 544. 
[10] Emmenegger C, Mauron P, Zuttel A, et al。Carbon nanotube synthe-sized on metallic substrates[J].Applied Surface Science, 2000，162 163:452 456。

[11] Ma Renzhi, Wei Bingqing, Xu Cailu, et al. [J]. Journal of Tsinghua University (Science and Technology Edition), 2000, 40(8): 7 10.

[12] Sun Xiaowei, Zhu Guofu. Electro-adsorption water treatment technology and equipment. Industrial Water Treatment, 2002, 22(8): 1 4.

[13] Sun Xiaowei, Zhu Guofu. Principle and composition of electro-adsorption water treatment technology (EST). Industrial water and wastewater, 2002, 33(4): 18 2.

[14] Chen Fuming. A new method for removing ions from water. [J]. Chemical Industry and Engineering Journal, 1999,50 (1): 114 117.

[15] Zhao Tingyue, Zhang Lingxiao, Zhang Aimin, et al. Electrosorption technology in water treatment applications. Shandong Water Resources, 2003, (2):35-36

[16] Liu Haijing, Zhang Hongtao, Sun Xiaowei. Experimental study on removal of ions in groundwater by electrosorption. Chinese Water Supply and Drainage, 2003,19(11):36 38

[17] Yang Yucheng, Sun Xiaowei, Zhu Guofu. EST technology and its residential drinking water equipment. Energy saving and environmental protection technology, 2002, 26 29 